\title{
Autosomal recessive infantile hypercalcemia
}

INSERM

\section{Source}

INSERM. (1999). Orphanet: an online rare disease and orphan drug data base. Autosomal recessive infantile hypercalcemia. ORPHA:300547

Autosomal recessive infantile hypercalcemia is a rare, genetic, phosphocalcic metabolism disorder characterized by early-onset hypercalcemia, hypophosphatemia, hypercalciuria, decreased intact parathyroid hormone serum levels and medullary nephrocalcinosis, typically manifesting with failure to thrive, hypotonia, vomiting, constipation and/or polyuria. 